\title{
Cytokine Adsorption in Septic Shock and Multiorgan Failure Following Major Obstetric Hemorrhage
}

\author{
Rachini Kishara Withanage, Nilmini Wijesuriya \\ Surgical Intensive Care Unit, Colombo North Teaching Hospital - Ragama, Sri Lanka.
}

Corresponding Author:
Dr. Rachini Kishara Withanage

Email: ork_168@yahoo.com

This is an Open Access article distributed under the terms of the Creative Commons Attribution License (creativecommons.org/ licenses/by/3.0).

Received Accepted

Published

November 4, 2016

March 3, 2017

March 30, 2017

\begin{abstract}
Background: Sepsis and septic shock is the second leading cause of mortality worldwide. It also significantly increases morbidity among intensive care unit patients. Case Report: We report on a 38 year old previously healthy patient who developed septic shock with multi-organ failure following a cardiac arrest due to massive obstetric hemorrhage. She had secondary hepatic impairment and acute kidney injury. Aggressive management of septic shock with cytokine adsorption supported recovery of failing organ systems. Conclusion: This case describes how cytokine filter was useful in the management of septic shock with multi-organ failure.
\end{abstract}

Keywords: Acute Kidney Injury, Cytokines, Multiple Organ Failure, Pregnancy, Sepsis, Septic Shock.

\section{Introduction}

A healthy patient who had massive obstetric hemorrhage leading to hypovolemic shock and cardiac arrest was successfully resuscitated but later developed septic shock and multi-organ failure. When all the other treatment modalities failed in her management, cytokine adsorption was used and the patient had complete recovery.

\section{Case Report}

A previously healthy 38 year old mother of three children was admitted with fever and watery vaginal discharge in her $18^{\text {th }}$ week of pregnancy. She has undergone cervical circlage two weeks back. In the hospital she developed severe vaginal bleeding and collapsed. She underwent examination under anesthesia and a partially aborted fetus was evacuated. As vaginal bleeding could not be controlled she underwent emergency total abdominal hysterectomy followed by bilateral iliac artery ligation. Pelvis was packed and abdomen was closed. She had massive blood transfusion and developed pulseless electrical activity twice, out of which she was successfully resuscitated.

She was admitted to the surgical intensive care unit (ICU) for further management. On admission to the ICU she was hypothermic and had signs of fluid overload. Echocardiography revealed good cardiac function. She was on inotropic support with adrenaline, noradrenaline and dobutamine. Adrenaline was tailed off. On the following day she underwent re-laparotomy for removal of abdominal packs. She developed atrial fibrillation intra-operatively and was treated with amiodarone. On the second post-operative day she developed signs of AKI (acute kidney injury) with rising serum creatinine levels. She became icteric and her liver enzymes started rising (ALT - 1086, AST - $9093 \mathrm{U} / \mathrm{L})$ with high bilirubin $(120 \mu \mathrm{mol} / \mathrm{L})$ and INR (2.5). Both acute kidney injury and acute liver failure were probably due to prolonged hypotension during resuscitation. She developed very high fever spikes $\left(105^{\circ} \mathrm{F}\right)$ on second ICU day with rising white blood cells and CRP (C-reactive 
protein). Initially all cultures were negative. She continued to be inotropic and ventilator dependent. Apart from above complications she also developed recurrent ventricular tachycardia with ischemic changes (troponin I positive), global myocardial hypo-kinesia and (DIC) disseminated intravascular coagulation.

Continuous renal replacement therapy (CVVHDF) was commenced on ICU day three with "Cytosorb" cytokine absorbent filter in addition to the hemofilter. Fever spikes, WBC counts and CRP started coming down. There was a dramatic decrease in AST, ALT and serum bilirubin levels [Fig.1,2]. Vasopressor and inotropic support was gradually tailed off over 10 days. But urine output did not improve and serum creatinine levels remained high. CRRT was continued for 10 days. Intermittent hemodialysis was started once she was hemodynamically stable. Then the platelet count started dropping and a diagnosis of heparin induced thrombocytopenia was made. Hemodialysis was continued without heparin. Acute liver failure improved with liver failure regime including $\mathrm{N}$-acetyl cysteine infusion, urosodil, rifaxamine and laxatives. Gradually she recovered from liver failure. As she was again having fever spikes, high WBC counts and continuously high output from abdominal drain, an exploratory laparotomy was done on ICU day nine. There was no intraabdominal collection. As it was difficult to wean her off from the ventilator, a tracheostomy was also done at the same time. Tracheostomy secretions, $\mathrm{CV}$ catheter tip were positive for Acinetobacter, which was only sensitive to polymixin. Later peripheral blood culture was positive for coagulase negative Streptococci and sputum for Coliform, which was only sensitive to polymyxin. Renal protective dose of polymyxin was continued for 10 days. Recurrent supraventricular tachycardia was treated with adenosine and amiodarone. During her ICU stay all fluid, vasopressors and inotropes administrations were guided by oesophageal Doppler cardiac output monitoring. All blood product transfusions were guided by rotational thromboelastometry (ROTEM).
She was gradually weaned off from the ventilator support. Nutrition support was given with total parenteral nutrition which was started on ICU day four as she did not tolerate enteral nutrition. Nasogastric feedings were started on ICU day 11 and TPN was gradually weaned off. She was treated in the intensive care unit for almost one month. With the all organs recovering, she continued to have low grade fever when she was discharged to ward. Urine culture was positive for coliform and was imipenem sensitive. Fever settled with treatment. Tracheostomy was closed. Urine output and renal functions gradually improved and hemodialysis was done thrice.

She developed urine incontinence while in the ward and a MRI scan showed a vesico-vaginal fistula. She was discharged home after 10 days of ward stay. At present she is back with her family after successful repair of the fistula.

\section{Discussion}

The maternal mortality rate in Sri Lanka is 30/10,000 live births [1]. Major obstetric hemorrhage is a leading cause of maternal mortality. This patient had major hemorrhage leading to cardiac arrest due to hypovolemic shock in early pregnancy. She required massive blood transfusion. Out of complications of massive blood transfusion she developed hypothermia, and transfusion related circulatory overload. She developed acute kidney injury and acute liver failure due to prolonged hypotension following cardiac arrest. On top of these acute insults she also developed septic shock which made the problems worse. Therefore aggressive management of sepsis was needed to support the failing organ systems.

She had a poor response to antibiotics and supportive therapy and continued to be septic with multiorgan failure. The overproduction of cytokines by the immune system, often called "cytokine storm" is a hallmark of septic shock. The "CytoSorb" is a medical device for extracorporeal cytokine removal. It is designed to remove other 
hydrophobic mid molecular substances like free hemoglobin, myoglobin, bilirubin and various bacterial endotoxins. "CytoSorb" is broadly indicated in any condition where cytokines are elevated. The technology is a biocompatible, highly porous polymer bead designed to capture and adsorb cytokines in the $10-50 \mathrm{kDa}$. It is compatible with almost all renal replacement systems and other extracorporeal blood pumps. It can be used as stand-alone therapy or in combination with RRT modes. The goal is to reduce toxic cytokine levels to prevent or mitigate organ failure and immune suppression, thereby improving clinical outcome.

"CytoSorb" has shown an overall improvement of MAP [2] with a rapid reduction in vasopressors dosage [2,3] in patients with severe sepsis/ SIRS, septic shock and multiorgan failure. Early start of therapy has shown better survival than later usage $[2,4]$. "CytoSorb" therapy was started early for our patient (ICU day three) and there was a dramatic reduction of liver enzymes after 24 hours of starting the therapy. Patients' clinical condition also improved significantly. Hepatic failure improved rapidly with combination of liver failure regime and "CytoSorb" therapy but the improvement of renal functions was slow. However renal functions recovered fully with CVVHDF and three cycles of hemodialysis. She developed a vesico - vaginal fistula which was repaired later. The patient is now fully recovered from all the complications she had.

This case builds upon how a patient having major obstetric hemorrhage leading to recurrent cardiac arrests complicated with ischemic hepatocellular and kidney injury with secondary sepsis was managed successfully with cytokine filter.

Contributors: Both authors have contributed to patient management and manuscript writing. RKW will act as guarantor. Both authors approved the final version of the manuscript.

Funding: None; Competing interests: None stated.

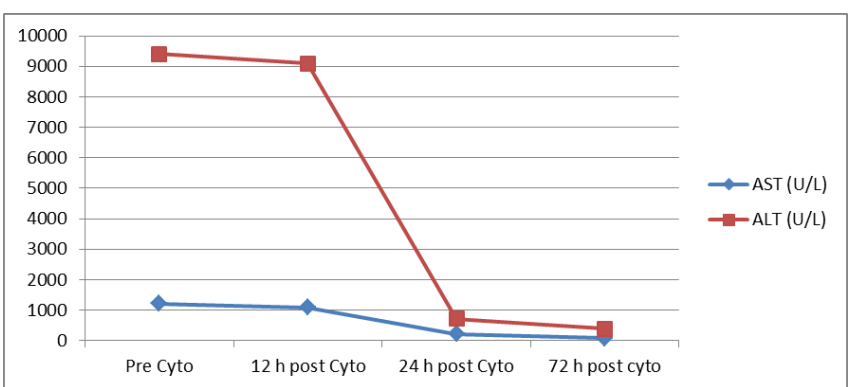

Fig.1: Change of liver enzyme levels with cytokine filter.

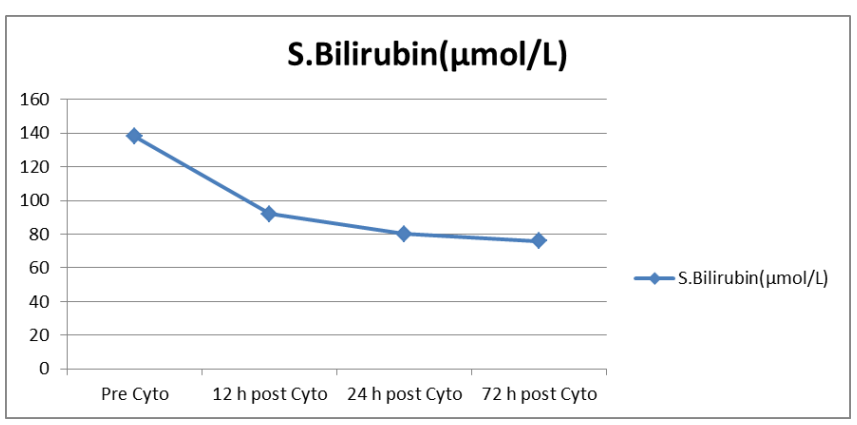

Fig.2: Change in serum bilirubin level with cytokine filter.

\section{References}

1. Maternal Mortality in 1990 - 2015: WHO, UNICEF, UNFPA, World Bank Group, and United Nations Population Division: Maternal Mortality Estimation Inter- Agency Group; Sri Lanka. Available at: http:// www.who.int/reproductivehealth/publications/ monitoring/maternal-mortality-2015/en/. Accessed on April 12, 2017.

2. Laddomada T, Doronzio A, Balicco B. Case series of patients with severe sepsis and septic shock treated with a new extracorporeal sorbent. Critical Care. 2016;20 (Suppl 2):193.

3. Kogelmann K, Druner M, Jarczak D. Case study of 8 patients with multiple organ failure treated additionally with cytosorbents haemadsorption as adjunctive therapy in septic shock and severe SIRS in cardiac failure. Infection. 2015;43 Suppl 1:1-73.

4. Sathe P, Sakhavalkar P, Kumar S, Choudhary S. Clinical experience in using a novel extracorporeal cytokine adsorption column for treatment of septic shock with multiorgan failure. Critical Care 2015;19 (Suppl 1):130. 